\title{
Novel Ocular Features in a Child with Marinesco-Sjögren Syndrome: Case Report and Literature Review
}

\author{
Syed M. Ali ${ }^{1}$ - Abdulrahman Gharkan ${ }^{2} \cdot$ Thomas M. Bosley $^{3} \cdot$ Igor Kozak $^{1}$
}

Accepted: 19 May 2021 / Published online: 29 May 2021

(C) The Author(s) 2021

\begin{abstract}
To report novel ocular findings in unique Marinesco-Sjögren syndrome (early-onset cataracts, cerebellar ataxia, and a progressive myopathy), review literature, and discuss causes of vision decrease in this rare entity. A 3-year-old girl diagnosed with Marinesco-Sjögren syndrome underwent uneventful cataract surgery in both eyes within 1 week of her 2nd birthday. This was exactly similar to her sister and other two related family members. Additionally, she had prominent corneal nerves and subtle corneal subepithelial deposits in both eyes. Cataracts are one of the primary diagnostic criteria for the Marinesco-Sjögren syndrome, and their early diagnosis and treatment are important for prevention of additional permanent visual loss. Prominent corneal nerves and mild corneal subepithelial defects are described in this report and may occasionally be helpful in making the diagnosis.
\end{abstract}

Keywords Marinesco-Sjögren syndrome $\cdot$ Pediatric cataract $\cdot$ Cornea $\cdot$ Prominent corneal nerves $\cdot$ Case report

\section{Background}

Marinesco-Sjögren syndrome (MSS) is a rare autosomal recessive condition characterized by early-onset cataracts, cerebellar ataxia, and a progressive myopathy [1]. The syndrome was first described by Marinesco et al. in a Romanian family [2] and further characterized by Sjogren [3] in families of Swedish origin. Previously used terms for this syndrome include Garland-Moorhouse syndrome, Marinesco-Garland syndrome, or hereditary oligophrenic cerebello-lental degeneration. In this report, we describe a 3-year-old child with the diagnosis of MSS confirmed both clinically and genetically who underwent cataract surgery at the age of 2 years. Three additional members of her extended family were also subsequently diagnosed with MSS. We review the literature of eye involvement in Marinesco-Sjögren syndrome and describe

Igor Kozak

igor.kozak@moorfields.ae

1 Moorfields Eye Hospitals UAE, Abu Dhabi, United Arab Emirates

2 Department of Pediatrics, Tawam Hospital, Al Ain, United Arab Emirates

3 Wilmer Eye Institute, Johns Hopkins University, Baltimore, MD, USA prominent corneal nerves and subtle corneal abnormalities not previously reported.

\section{Case Presentation}

The proband was the product of a normal pregnancy and a spontaneous vaginal delivery at 40 weeks gestation to consanguineous parents. The child had normal birth weight, length, and head circumference. Development seemed normal over the first 3 months after delivery, although she did have an unusual number of bowel movements, reaching up to 30 times a day with small amounts of stools. After 3 months of age, she developed an excessive startle response after sudden or loud noises. Subtle spontaneous motor movements started to appear around the age of 6 months, including writhing movements of her feet, titubation of the head, and horizontal pendular nystagmus.

She had developmental delay of motor functions along with social, psychomotor, and speech delay. The features of cerebellar dysfunction became more evident after 1 year of age, including hypotonia, tremor, ataxia, dysmetria, dysdiadochokinesis, and very limited, largely unintelligible speech. The clinical presentation was thought to strongly suggest a diagnosis of autosomal recessive spinocerebellar ataxia. 
Whole exome sequencing (CentoXome GOLD®), Rostock, Germany) detected a novel homozygous variant in the SIL1 gene (c.947T $>$ G p.Leu316Arg in exon 10) that is likely pathologic. The SIL 1 gene is commonly associated with autosomal recessive Marinesco-Sjögren syndrome.

Her general and neurologic exams were followed monthly by her father, who is a pediatrician, and 1 week prior to her second birthday, he noted that she had an abnormal red reflex OS>OD. Examination at Moorfields Eye Hospital Abu Dhabi revealed bilateral cataracts (Figs. 1 and 2) for which she underwent uneventful cataract extraction with implantation of intraocular lenses. The remainder of her ophthalmologic exam was grossly normal, including normal retinae and posterior poles. However, she had faint, non-specific linear subepithelial corneal opacities with few sub-epithelial dots (Fig. 3) and bilateral prominent corneal nerves (Figs. 4 and 5). She had a 25 diopter comitant esotropia and fixated with her right eye. She had no obvious nystagmus at the time of this exam.

At 3 years of age, she weighed $9.8 \mathrm{~kg}$ and was $90 \mathrm{~cm}$ tall with a head circumference of $45 \mathrm{~cm}$ (25th percentile). She sat without support, but she could not stand. She could use a baby walker and could slide around on her back using her legs to push herself forward. She could reach for nearby objects despite an upper extremity tremor and could pick up small objects off the ground. She could see and respond to screens of a mobile phone. Her binocular visual acuity was $0.6 \log$ MAR (the equivalent of 20/80) after cataract surgery. She was under the care of a pediatric neurologist and continued visual rehabilitation.

The proband's sister was 16 years old when first evaluated at Moorfields Hospital Abu Dhabi and had similar systemic, ophthalmologic, and neurologic signs and symptoms. She had short stature, delayed puberty, and a clinical picture of cerebellar malfunction with significant ataxia. Like her sister, she had developed bilateral cataracts a week before her second birthday and underwent cataract surgery expeditiously. Her

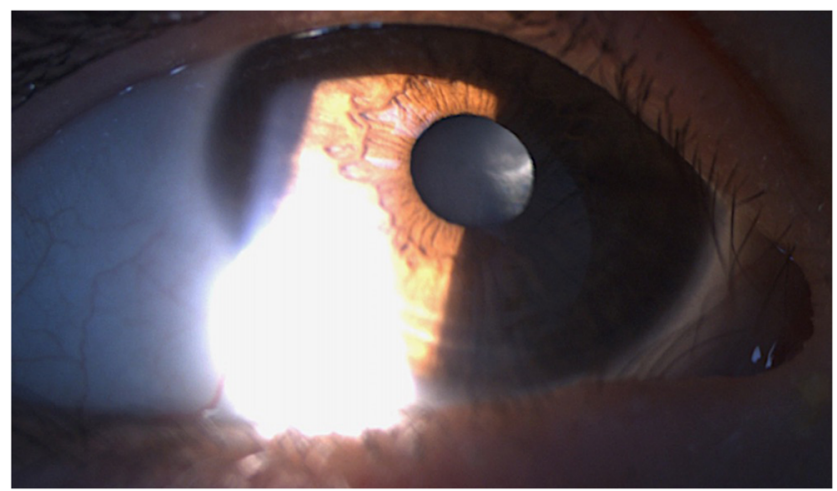

Fig. 1 A slit-lamp photograph of a 2-year-old girl with MarinescoSjögren syndrome (MSS) showing visually significant cataract in her right eye

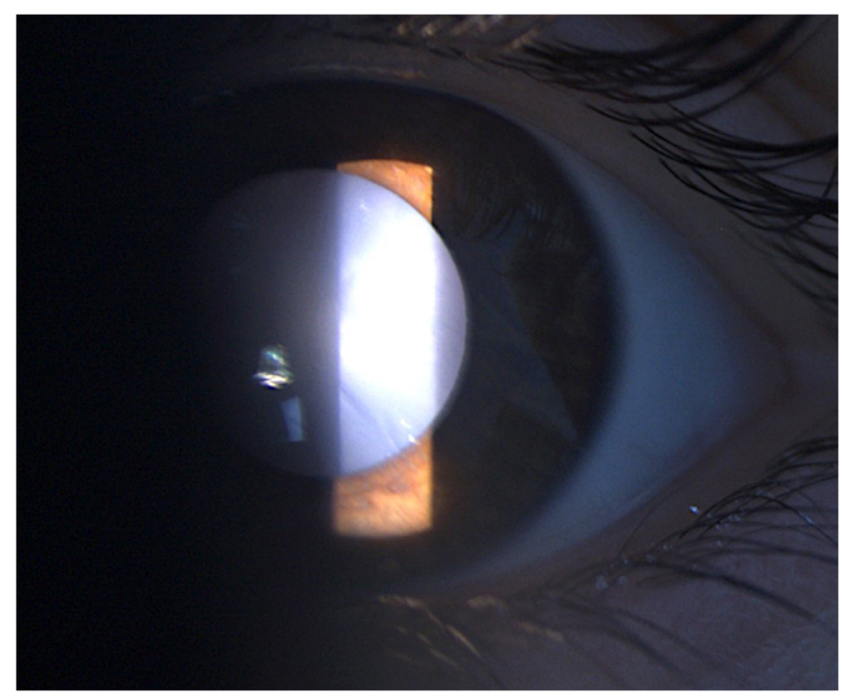

Fig. 2 A slit-lamp photograph of a 2-year-old girl with MarinescoSjögren syndrome (MSS) showing visually significant cataract in her left eye

examination revealed bilateral pseudophakia with high myopia, amblyopia, and prominent corneal nerves. Two male cousins of a consanguineous marriage on the maternal side of the family had the same signs and symptoms with the same chronological appearance and course of bilateral cataracts within weeks of their second birthday. The older cousin was 26 years old when his family was contacted and had been immobile and bed ridden for the previous 2 years; the younger boy was 18 years old with similar disabilities. These individuals lived at some distance and details of current ophthalmologic examinations were not available.

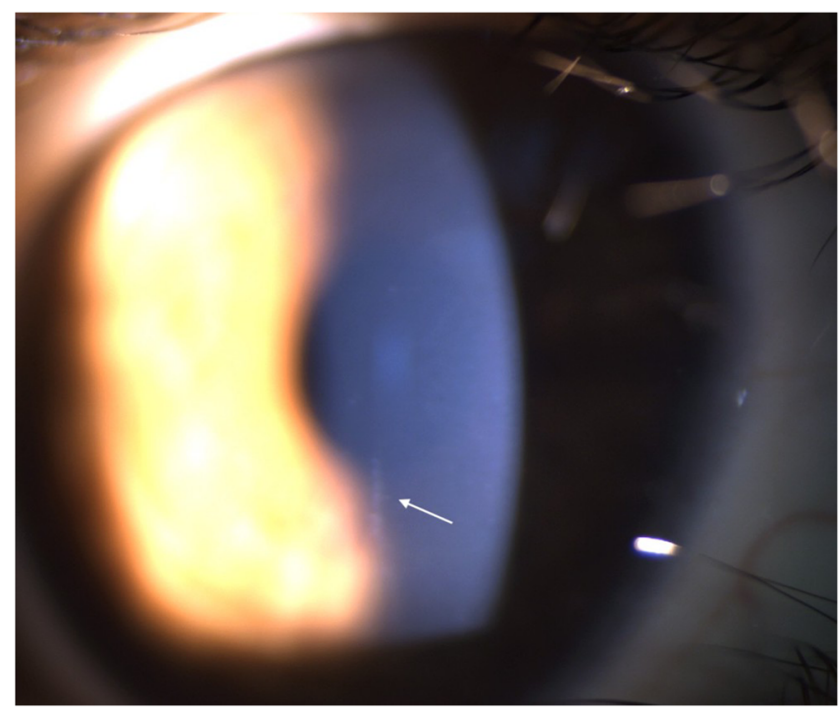

Fig. 3 A slit-lamp photograph of a 2-year-old girl with MarinescoSjögren syndrome (MSS) showing a non-specific linear subepithelial faint corneal opacities (white arrow) with few sub-epithelial dots 


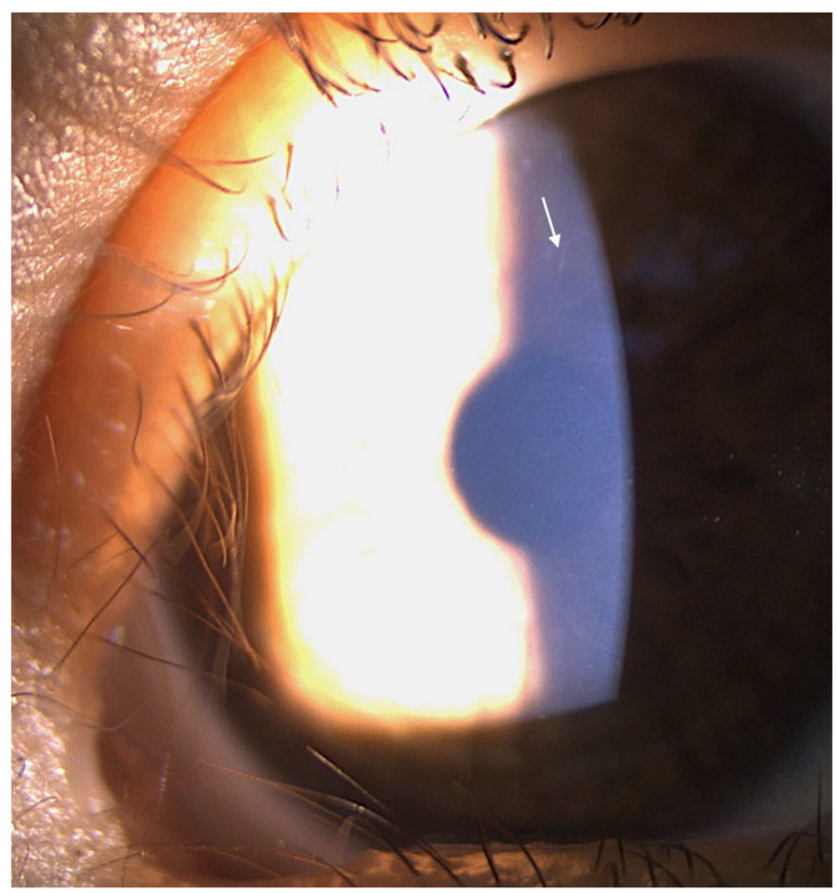

Fig. 4 A slit-lamp photograph of a left eye of 2-year-old girl with Marinesco-Sjögren syndrome (MSS) showing prominent corneal nerves (white arrow)

\section{Discussion and Conclusions}

The cardinal clinical features of MSS include bilateral cataracts, cerebellar ataxia, psychomotor delay, and progressive muscle weakness. Additional clinical features that have been described include short stature and other skeletal abnormalities [4], hypogonadism [5], strabismus [6], microcephaly [7],

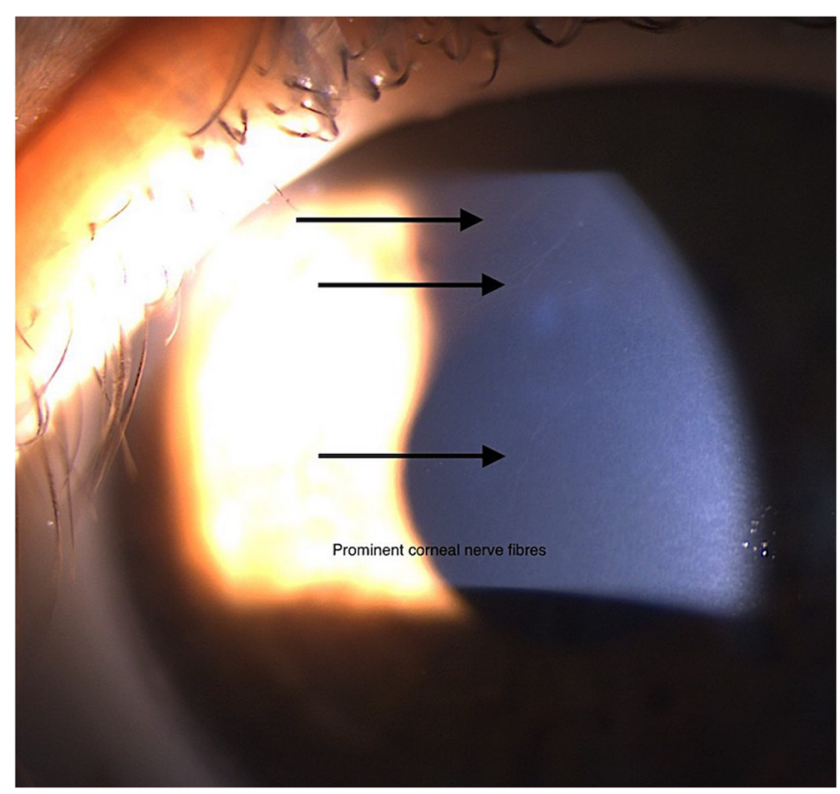

Fig. 5 A slit-lamp photograph of a right eye of 2-year-old girl with Marinesco-Sjögren syndrome (MSS) showing prominent corneal nerves (black arrows) and nystagmus [8]. Clinical diagnosis is complicated by the fact that these characteristics are variable both in occurrence and in time of presentation. For example, cataracts were initially described as congenital [3] but more recently have been recognized to appear bilaterally during early childhood and to progress rapidly $[9,10]$. Hypotonia is often present in early infancy, while weakness and cerebellar signs usually appear during childhood and progress into adult life [9]. Neuroimaging can be diagnostically helpful when cerebellar atrophy (particularly the vermis) and T2-hyperintense cerebellar cortex are apparent [11]. Electron-microscopic ultrastructural changes on muscle biopsy are thought to be pathognomonic of MSS [12].

Cataracts are one of the defining features of MSS [1-3] so that the clinical diagnosis is often difficult to confirm if the patient is evaluated prior to the appearance of cataracts [10]. The mean age at onset of cataracts is around 3.5 years [6], and cataracts typically progress rapidly and require lens extraction in the first decade of life $[6,12]$. Interestingly, all affected children in this extended family developed bilateral cataracts within weeks of their second birthday, an observation not described previously in MSS. This medically sophisticated family admitted, however, that following the onset of cataracts in the first affected family member at the age of 2 years (the older male cousin), other potentially affected family members were regularly examined for cataract formation.

A number of other ophthalmologic abnormalities have been described at least intermittently in MSS so that a careful ophthalmologic examination may help in the diagnosis. Perhaps most importantly, children of the family reported here, similar to other reported individuals [6], had modestly reduced visual acuity bilaterally (roughly 20/80 binocular acuity in the proband after cataract surgery). Chronically reduced visual acuity might, in turn, be the cause of bilateral amblyopia with subsequent horizontal pendular nystagmus (looking like infantile nystagmus) [9] and modest comitant strabismus without gross limitation of ocular motility (roughly equally divided between esotropia and exotropia) [6] that have been reported regularly in MSS patients. Bilateral optic atrophy has also been reported [13].

The etiology of reduced visual acuity in both eyes was not clear in this family. Cataracts were modestly dense in the proband at the time of cataract surgery, but the surgery was done quickly, and neither the timing nor the results of the surgery explain subsequently reduced visual acuity. The proband had no obvious retinal or optic disk abnormality and no major malformation of the anterior segment in either eye. Accurate refraction was difficult in this child with both cognitive and motor limitations, but a major refractive error was not noted here and has not been reported previously. Iris colobomas have been described [14], but corneal opacification has not. The proband had clear corneas but did have the novel observation of prominent corneal nerves, 
which are seen in a number of other genetic ocular and systemic conditions without effect on vision [15-17]. Nonspecific subepithelial corneal opacities were also noted in the proband. They were subtle and of unknown origin; however, Zimmer et al. [18] studied light and electron microscopy of conjunctiva in six patients with MSS, and biopsies revealed a marked increase in the number of lysosomes in fibroblasts.

The mutated gene causing MSS is usually SIL1 (Gene ID:64374) on chromosome 5q31.2 [7, 19], which creates an evolutionarily conserved, widely expressed glycoprotein known as SIL1 or BAP [20]. It interacts strongly with the HSP70 family of proteins that are highly conserved molecular chaperones known to assist in stabilization and folding of newly synthesized polypeptides [19], translocation of proteins across the endoplasmic reticulum membrane, and regulation of calcium homeostasis. The diagnosis of MSS should be considered in an individual with compatible clinical abnormalities and can be confirmed by the presence of biallelic pathogenic variants of SIL1 identified on molecular genetic testing $[7,19]$. Molecular genetic testing is rapidly becoming less expensive and easier to obtain and, depending on the evaluating institution and the clinical picture of a child with possible MSS, can include single-gene testing for SIL1, a multigene panel, or comprehensive genomic testing such as exome or genome sequencing (as done here). Most MSSassociated SIL1 pathogenic variants predict protein truncation likely to render the protein non-functional.

The major differential diagnosis for MSS includes the autosomal recessive syndrome defined by congenital cataracts, facial dysmorphism and neuropathy (CCFDN) caused by a defect in the CTDP1 gene [OMIM 604168] [21, 22]. Other potential diagnoses include autosomal dominant ITM2B-related cerebral amyloid angiopathy [OMIM 117300] [23], autosomal recessive INPP5K-related muscular dystrophy with congenital cataracts [OMIM 617404] [24], and autosomal recessive GBA2-related Marinesco-Sjögren syndrome-like disorder [OMIM 609471] [25]. Individuals initially described as having another Marinesco-Sjögren-like syndrome (also called ataxia-juvenile cataract-myopathy-intellectual disability [OMIM 248810]) were later found to have classic MSS, resulting in discontinuation of this OMIM entry [9]. A subgroup of patients with clinical characteristics typical of MSS do not have an identified SIL1 point mutation, suggesting alternative genetic mechanisms or locus heterogeneity in some patients $[7,26]$.

In conclusion, cataracts are one of the primary diagnostic criteria for the Marinesco-Sjögren syndrome, and their early diagnosis and treatment are important for prevention of additional permanent visual loss. Prominent corneal nerves and mild corneal subepithelial defects are described in this report and may occasionally be helpful in making the diagnosis. Other ophthalmologic signs include nystagmus, strabismus, and possibly amblyopia, all of which may be secondary to moderately poor vision OU from early childhood of unclear etiology. Other ophthalmologic abnormalities rarely noted include optic atrophy and iris colobomas.

Abbreviations MSS, Marinesco-Sjögren syndrome; $O D$, oculus dexter (right eye); $O S$, oculus sinister (left eye); $\log M A R$, logarithm of minimal angle of resolution

Author contribution Data collection (SA, AG, TMB, IK), drafting of the manuscript (TMB, IK), review of the manuscript (SA, AG, TMB, IK). All authors have read and approved the manuscript.

Data availability Yes

Code availability None

\section{Declarations}

Competing interests The authors declare no competing interests.

Ethics approval Waived by Research and Ethics Committee of the Moorfields Eye Hospital Abu Dhabi, U.A.E.

Consent to participate Written consent has been obtained from parents of the participant and is available for review by the Editor of this journal.

Consent for publication Yes

Open Access This article is licensed under a Creative Commons Attribution 4.0 International License, which permits use, sharing, adaptation, distribution and reproduction in any medium or format, as long as you give appropriate credit to the original author(s) and the source, provide a link to the Creative Commons licence, and indicate if changes were made. The images or other third party material in this article are included in the article's Creative Commons licence, unless indicated otherwise in a credit line to the material. If material is not included in the article's Creative Commons licence and your intended use is not permitted by statutory regulation or exceeds the permitted use, you will need to obtain permission directly from the copyright holder. To view a copy of this licence, visit http://creativecommons.org/licenses/by/4.0/.

\section{References}

1. Herva R, von Wendt L, von Wendt G, Saukkonen AL, Leisti J, Dubowitz V. A syndrome with juvenile cataract, cerebellar atrophy, mental retardation and myopathy. Neuropediatrics. 1987;18(3): 164-9.

2. Marinesco G, Draganesco S, Vasiliu D. Nouvelle maladie familiale characterise par une cataracte congenitale et un artet du development somato-neuro-psychique. L'encephale. 1931;26:97-109.

3. Sjogren T. Hereditary congenital spinocerebellar ataxia accompanied by congenital cataract and oligophrenia; a genetic and clinical investigation. Confin Neurol. 1950;10(5):293-308.

4. Brogdon BG, Snow RD, Williams JP. Skeletal findings in Marinesco-Sjogren syndrome. Skelet Radiol. 1996;25(5):461-5. 
5. Skre H, Berg K. Linkage studies on Marinesco-Sjogren syndrome and hypergonadotropic hypogonadism. Clin Genet. 1977;11(1): 57-66.

6. Goto M, Okada M, Komaki H, Sugai K, Sasaki M, Noguchi S, et al. A nationwide survey on Marinesco-Sjogren syndrome in Japan. Orphanet J Rare Dis. 2014;9:58.

7. Senderek J, Krieger M, Stendel C, Bergmann C, Moser M, Breitbach-Faller N, et al. Mutations in SIL1 cause MarinescoSjogren syndrome, a cerebellar ataxia with cataract and myopathy. Nat Genet. 2005;37(12):1312-4.

8. Lagier-Tourenne C, Chaigne D, Gong J, Flori J, Mohr M, Ruh D, et al. Linkage to 18qter differentiates two clinically overlapping syndromes: congenital cataracts-facial dysmorphism-neuropathy (CCFDN) syndrome and Marinesco-Sjogren syndrome. J Med Genet. 2002;39(11):838-43.

9. Anttonen AK. Marinesco-Sjogren Syndrome. In: GeneReviews $((R))$. edn. Edited by Adam MP, Ardinger HH, Pagon RA, Wallace SE, Bean LJH, Stephens K, Amemiya A. Seattle (WA); 1993.

10. Ishikawa T, Kitoh H, Awaya A, Nonaka I. Rapid cataract formation in Marinesco-Sjogren syndrome. Pediatr Neurol. 1993;9(5):407-8.

11. Harting I, Blaschek A, Wolf NI, Seitz A, Haupt M, Goebel HH, et al. T2-hyperintense cerebellar cortex in Marinesco-Sjogren syndrome. Neurology. 2004;63(12):2448-9.

12. Krieger M, Roos A, Stendel C, Claeys KG, Sonmez FM, Baudis M, et al. SIL1 mutations and clinical spectrum in patients with Marinesco-Sjogren syndrome. Brain. 2013;136(Pt 12):3634- 44.

13. Dotti MT, Bardelli AM, De Stefano N, Federico A, Malandrini A, Vanni M, et al. Optic atrophy in Marinesco-Sjogren syndrome: an additional ocular feature. Report of three cases in two families. Ophthalmic Paediatr Genet. 1993;14(1):5-7.

14. Ezgu F, Krejci P, Li S, de Sousa C, Graham JM Jr, Hansmann I, et al. Phenotype-genotype correlations in patients with MarinescoSjogren syndrome. Clin Genet. 2014;86(1):74-84.

15. Al-Faky YH, Bosley TM, Al-Turki T, Salih MA, Abu-Amero KK, Alsuhaibani AH. Prominent corneal nerves: a novel sign of lipoid proteinosis. Br J Ophthalmol. 2012;96(7):935-40.

16. Chamney S, O'Neill M, George S. Prominent corneal nerves. J Pediatr. 2014;165(3):636.

17. Kinoshita S, Tanaka F, Ohashi Y, Ikeda M, Takai S. Incidence of prominent corneal nerves in multiple endocrine neoplasia type 2A. Am J Ophthalmol. 1991;111(3):307-11.
18. Zimmer C, Gosztonyi G, Cervos-Navarro J, von Moers A, Schroder JM. Neuropathy with lysosomal changes in Marinesco-Sjogren syndrome: fine structural findings in skeletal muscle and conjunctiva. Neuropediatrics. 1992;23(6):329-35.

19. Anttonen AK, Mahjneh I, Hamalainen RH, Lagier-Tourenne C, Kopra O, Waris L, et al. The gene disrupted in MarinescoSjogren syndrome encodes SIL1, an HSPA5 cochaperone. Nat Genet. 2005;37(12):1309-11.

20. Chung KT, Shen Y, Hendershot LM. BAP, a mammalian BiPassociated protein, is a nucleotide exchange factor that regulates the ATPase activity of BiP. J Biol Chem. 2002;277(49):47557-63.

21. Tournev I, Kalaydjieva L, Youl B, Ishpekova B, Guergueltcheva V, Kamenov O, et al. Congenital cataracts facial dysmorphism neuropathy syndrome, a novel complex genetic disease in Balkan Gypsies: clinical and electrophysiological observations. Ann Neurol. 1999;45(6):742-50.

22. Tournev I, King RH, Workman J, Nourallah M, Muddle JR, Kalaydjieva L, et al. Peripheral nerve abnormalities in the congenital cataracts facial dysmorphism neuropathy $(\mathrm{CCFDN})$ syndrome. Acta Neuropathol. 1999;98(2):165-70.

23. Stromgren E, Dalby A, Dalby MA, Ranheim B. Cataract, deafness, cerebellar ataxia, psychosis and dementia-a new syndrome. Acta Neurol Scand. 1970;46(S43):261-2.

24. Osborn DPS, Pond HL, Mazaheri N, Dejardin J, Munn CJ, Mushref $\mathrm{K}$, et al. Mutations in INPP5K cause a form of congenital muscular dystrophy overlapping Marinesco-Sjogren syndrome and dystroglycanopathy. Am J Hum Genet. 2017;100(3):537-45.

25. Hammer MB, Eleuch-Fayache G, Schottlaender LV, Nehdi H, Gibbs JR, Arepalli SK, et al. Mutations in GBA2 cause autosomal-recessive cerebellar ataxia with spasticity. Am J Hum Genet. 2013;92(2):245-51.

26. Anttonen AK, Siintola E, Tranebjaerg L, Iwata NK, Bijlsma EK, Meguro H, et al. Novel SIL1 mutations and exclusion of functional candidate genes in Marinesco-Sjogren syndrome. Eur J Hum Genet. 2008;16(8):961-9.

Publisher's Note Springer Nature remains neutral with regard to jurisdictional claims in published maps and institutional affiliations. 